\title{
Simulation Analysis for in-Line Sorting-and-Washing of Reusable Pallets: A Case Study
}

\author{
Yeongmin YUN, Junjae CHAE*
}

\begin{abstract}
In this study, a system constituting an integral part of a continuous sequence of operations or machines in a line is presented as a form of "in-line" system. Due to the sequential nature of the production line, the throughput rate of the line depends on the slowest process. This paper presents one example of an in-line system that is used for pallet re-use by sorting, washing, and drying in a continuous processing line. Basically, a well-structured in-line system provides high throughput because of the non-stop flow of materials in the system. However, there is a hidden loss in the system efficiency. The object of this paper is to evaluate some of the possible alternatives to solve these hidden inefficiency problems and to improve throughputs through simulated models. The three outcomes from this simulation indicate that exchanging the robotic arm with a sorter and adding additional spin-drying machines could reduce overhead costs and the average waiting time for the spin-drying machine and improve the utilization of the resources in the washing process.
\end{abstract}

Keywords: continuous material flow system; in-line system; pallet reuse

\section{INTRODUCTION}

An "in-line" system is a type of system which constitutes a continuous sequence of operations using machines, robots, or humans arranged in a straight line. In the manufacturing industry, the in-line system is referred to as the transfer line where materials flow from one workstation to the next in a sequential manner [1]. Due to the sequential nature of the production line, the throughput rate of the line is dependent on the slowest process [2]. An in-line system is one example of the fixed automation systems.

The production line layout is critical in the overall efficiency of the manufacturing system. Among the many different manufacturing system layouts, in-line layouts, which are a kind of transfer lines, are used for automated high-volume production. In highly automated lines, each machine's processing rates are matched in order that a buffer storage between machines is not necessitated. However, the major drawbacks of such a system include very high equipment cost, lack of flexibility in layout and the number of possible products that can be manufactured. Since the structure of the transfer line only consists of one line with the equipment arranged sequentially, the ways to improve the transfer line are very limited. Such approaches to improve efficiency include substituting inefficient equipment with more efficient equipment or changing the process order.

Since materials move from one machine to the next in a sequential processing line in an in-line system, the in-line system is like a material flow system. Improving the material flow system means reducing the overall flow of the system. One way of minimizing the total flow is the work simplification of the material flow [1]. The work simplification method of material flow involves the following steps: the first is to remove the intermediary steps in the flow by delivering materials directly to the usage point; the second is to minimize the number of flows between two sequential points by using as few movements as possible; the third is to arbitrarily combine flows and operations at a point where it is possible; and the fourth is to eliminate unnecessary movements of materials by reducing the number of manufacturing steps.
Meanwhile, in the pallet pool system, a company rents pallets of which the size and dimensions are standardized from a shared pool instead of making or buying individualized pallets $[3,4]$. The pallet pooling company ensures that the supply of pallets, stored in their warehouse is in good repair and ready for use and delivery as the customer needs them. In addition, the pallet company will also arrange for the pallets to be picked up and returned to the warehouse or to deliver the unused pallets to other customers. Moreover, a pallet pooling service provider deals with the reverse logistics by recovering these pallets for re-use from the customer or from other manufacturers.

Generally, the flow in a pallet pooling system is as follows. First, delivery trucks go to the pallet warehouse where pallets that are required are loaded onto the delivery trucks. Next, the pallets are delivered to and unloaded for the customer. The customer's finished products are placed on the pallets and sent to distribution terminals. At the distribution terminals, the products on the pallets are sorted and shipped to regional service centers, where the products are unloaded from the pallets. Finally, the empty pallets are picked up and returned to the pallet warehouses.

Some of the used pallets are discarded, while others need to be cleaned. Therefore, pallets which are returned to the pallet pooling company's warehouse need to be sorted. After sorted, the damaged pallets are discarded, and good ones are washed for re-use. Hence, at the warehouses of the pallet pooling service provider, there are processes which sort and wash pallets. These processes can be positioned in separated locations, and also, they are connected in sequential order as part of the material flow system.

Especially, a pallet polling company in South Korea has a warehouse where there is an in-line system which starts with the sorting process and ends with washing and drying process. This in-line system is the second case of pallet sorting and washing processes as mentioned above. Even though the transfer line or in-line system has the disadvantage of high equipment cost, this flow line uses a robotic arm to sort pallets before the pallets move to the washing process because using the robotic arm typically has led to higher fixed overhead costs in the in-line system. Further, there have been unnecessary flows of pallet and queues, and they have increased the material handling costs of the system. Therefore, this paper builds a model of the 
existing system and sets out to improve the existing model by seeking to improve upon these inefficiencies and/or lowering fixed overhead costs. The current palletizing process is replaced with a sorter on the conveyor. The order of the process for sorting the class of pallets that need to be washed is changed after the washing process. In other words, the pallets are divided in two groups before the washing process. A group is for reusable pallets which are sent to washing process and this group is classified into two classes based on its reusability after the washing process. Another group is for the pallets which need ether to be reconstructed or to be discarded and the pallets in this group are not sent to the washing process.

This paper is organized as follows. The section 2 provides reviews of the literature related to improving the material flow system through the simulation approach and using AutoMod simulation software. Section 3 describes the system of the case study, which includes sequences of each process, main assumptions, and problems in this system in detail. Section 4 explains the methodology of this research. In Section 5, the overall contents of the simulation model and the experiment are elaborated upon. The data collection and analysis for this research are discussed, and this section describes the simulation model of the existing system, model assumptions, the alternative scenarios to ameliorate the current in-line system, and experimental design in detail. Section 6 discusses the results of experiments relative to these alternative scenarios. Section 7 provides the conclusion of this paper with a summary of the research, limitations and implications.

\section{LITERATURE REVIEW}

A manufacturing flow line is viewed as a sequence of workstations separated by buffers of finite capacity [5]. Materials pass through a sequential system and a specific operation is performed at each station. Each material flows from outside the system to the first machine, and on to various machines until the last machine is reached. Afterwards, the material exits the system. Each product takes certain time, called the processing time, to be processed on a given machine. A production line where processing times of all machines are the same is referred to as a homogeneous line [6]. But in general, the processing times on different machines are not equal [7]. This production line is referred to as a nonhomogeneous line.

Numerous studies in the literature have been published on developing analytical models of production systems. We understand the effects of system features such as processing rates and buffer capacities, processing time variability, machine reliability, and material flow on system performance through analytical models of production systems. These models enable us to estimate the performance of a material flow system and optimize design parameters of the system [8].

In the past, most studies have assumed homogeneous production lines to be discrete flow of materials, which is associated with Markov chain models $[9,10]$ and the lines are restricted to homogeneous lines. Such models' production rate is calculated from the steady-state probabilities. The production rate is the amount of materials produced per unit time for long run. Yeralan and
Muth presented a general state nonhomogeneous discrete flow model [10].

The studies of models with continuous materials flow have been continuous, although few. There are several studies that analyse models of a two-station continuous material system which has a finite buffer in the production lines. Both Zimmern [11] and Sevast'yanov [12] analyzed a two-machine continuous material flow system assuming that the distributions of the failure and repair times are exponential. Wijngaard [13] presented the production rate of a production line with continuous materials flow as a closed-form solution. Murphy [14] went beyond a recursive equation to evaluate the expected improvement in the production rate using additional buffers. Yeralan et al. [15] developed a two-station continuous materials flow production line model whose behavior is described in a closed form.

There are also several studies that analyze models using multiple states in production systems. Dallery [16] analyzed mainly with failure and repair times in stochastic models of production lines which have more than two states. Levantesi et al. [17] evaluated the performance of continuous manufacturing systems with machines which have different processing times and several failure modes. Kim and Gershwin [18] studied a continuous materials flow model of manufacturing systems with single and two dual machines. Colledani and Gershwin [19] analyzed a multi-stage fluid flow system with machines characterized by continuous discrete state Markov chain of general complexity and finite buffer capacity. Fleuren et al. [20] analyzed performance of exponential multi-server production lines with fluid flow and finite buffers.

Various problems such as queues caused by difference in speed of machines make the system inefficient. For example, station breakdown is a major cause of line inefficiency [21]. Therefore, there are several studies to be found in the literature related to the improvement of the material flow system or manufacturing lines. In trying to analyze material's flows and solve existing problems, also because of the complexity of the material flow system, a simulation approach has been considered a general method. Rohleder et al. [22] found an orthopaedic outpatient clinic's improvement alternatives optimized staffing levels, better patient scheduling, and attention on staff reaching immediately by modeling the existing system, and they analyzed the result taken before and after the implementation of changes. Huang et al. [23] proposed a systematic methodology for productivity measurement and analysis at the plant level. Through the simulation approach employing this methodology, they improved the manufacturing line in the case study and effectively demonstrated that their techniques improve manufacturing productivity. Kamrani et al. [24] simulated the traffic on the network of two junctions located at a case study city during rush hours and analyzed its constrictions and suggested alternatives to improve on these constrictions.

This study is similar to the above cited papers in terms of continuous materials flow process management. This inline system is the case of continuous flow of discrete material and the simulation model can provide tools for reasonable approach when there are some changes in such system. 


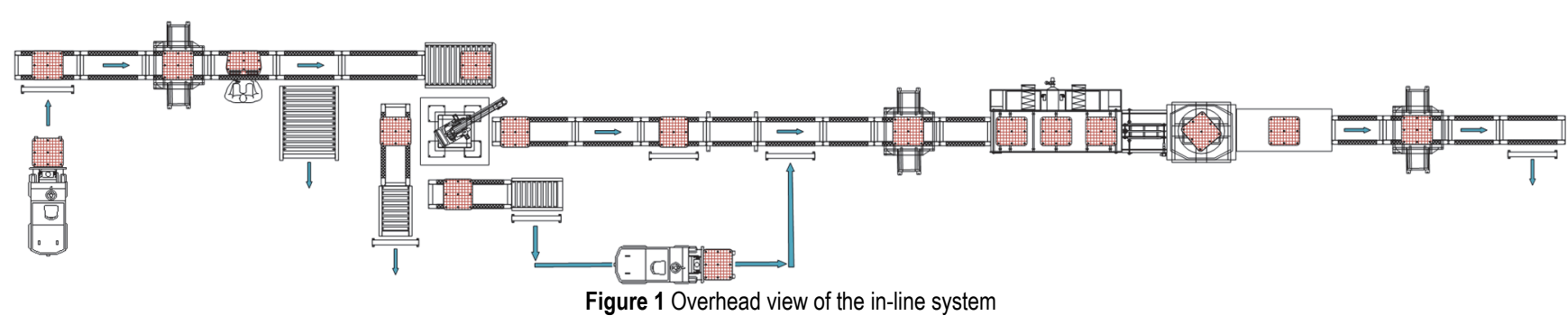

As shown in Fig. 1, we can monitor the material move and change through the tools and directly apply any change to the model and analyse if there is an improvement.

\section{PROBLEM DESCRIPTION}

This paper focuses on improving the in-line system which is a simple one-line queueing network and starts from sorting process to washing and drying process at the warehouse of the case study organization which is a pallet polling company (see Fig. 1).

After a retrieving truck collects the used pallets and returns them to the warehouse of the case study factory, the pallets are unloaded from the retrieving truck and placed in the warehouse using forklifts. The collected pallets, which are in stacks of 16, are divided into four classes according to the state of the pallet: A, B, C or D. It means that A class is the best state and D class is the worst. However, if a pallet is hard to wash because of heavy contamination, it is also sorted as D class. The pallets of A and B classes are delivered to general companies. Due to heavily damaged state, a C class pallet is delivered to a special company and does not require cleaning. D class pallets are not washed because they are subject to disposal.

\subsection{Sequence of Sorting Process}

The sorting process of the in-line system is shown in Fig. 2. Initially, a worker, using a forklift puts a stack of unsorted pallets onto the chain conveyor. Once the first pile enters the unstacker, a second stack is placed on the conveyor. The remaining pile of pallets is loaded and moves to a waiting point until all pallets of the previous pile are unstacked and have exited the unstacker. When a pile of pallets comes in, the unstacker separates the pallets one by one. Each time the unstacker separates one pallet, unstacking time is required. The removed pallet moves to a point where a worker determines the class of the pallet. At this point, however, the worker not only determines the class, but also removes contaminants. If the previous pallet is contaminated, additional time is required for the worker to decontaminate the pallet which in turn increases the time the next pallet remains in the unstacker.

The sorted pallet advances to the next point that determines whether or not it is class D. Thus, when the pallet reaches this point, any $\mathrm{D}$ class pallet is removed from the in-line system. Otherwise, the pallet goes to a waiting point to be sorted by a robotic arm.

The remaining pallets proceed to the next waiting point while the robotic arm which can rotate $360^{\circ}$, sorts these pallets into either A, B, or C class. Once a pallet is sorted, the robotic arm repeats the process for the remaining pallets. If the pallet is A, then the body of the robot arm turns $180^{\circ}$ clockwise. If the pallet is $\mathrm{B}$, then the robot arm turns $90^{\circ}$ clockwise. If the pallet is $\mathrm{C}$, then the robot arm turns $90^{\circ}$ counter clockwise. Pallets of each class continue to be stacked until the number of pallets in a stack meets a batch size (lot size). When the stack of pallets reaches the batch, the A stack is stored on the floor until a worker puts the pallets one by one on a chain conveyor of the washing process with a forklift. The B stack goes through the washing process and the $\mathrm{C}$ stack is removed from the inline system like D pallets.

\subsection{Sequence of Washing Process}

The pallet washing process can be divided into three stages: washing, spin-drying and drying. In this case study, there are three washing machines, one spin-drying machine, and two drying machines, as shown in Fig. 3. All stages of the washing process are carried out while pallets pass through the machines of each sub-process on the conveyor. Conveyor system of washing stage is constituted of a number of individual conveyors, not just one conveyor. Therefore, while washing, only one pallet can enter a washing machine. In other words, the next pallet cannot enter the machine before a pallet passes completely through. In spin-drying, a spin-drying machine spin-dries a pallet by spinning it for a second.

Only A and B pallets can go through the washing process. Stacks of B move to the washing process from the sorting process on the conveyor. Meanwhile, stacks of A are input at a point in front of an unstacker which is part of the washing process, when there is no B stacks in queue. The unstacker in the washing process operates like the one in the sorting process. Each pallet separated from unstacker goes into the washing machine. Pallets are washed while passing through the wash machines arranged in a row. The washed pallets are sent to the spin-drying part. In this part, pallets can enter the spin-drying machine when the spinning has been completed for the previous pallet. When the spin-dried pallets move to the drying part, two drying machines carry the drying work. Pallets are passed through the drying machines like the washing stage. The dried pallets are sent to a pallet stacker. In the pallet stacker, pallets are stacked until the number of stacked pallets are the batch size which is the same size when entering the sorting process on the conveyor. The stack of pallets with the required batch size then exits the in-line system.

\subsection{Problems in Current System}

There are two problems with the mentioned in-line system: first, the usage of a robotic arm in the sorting process, and the second is a queue in front of the spindrying machine. 


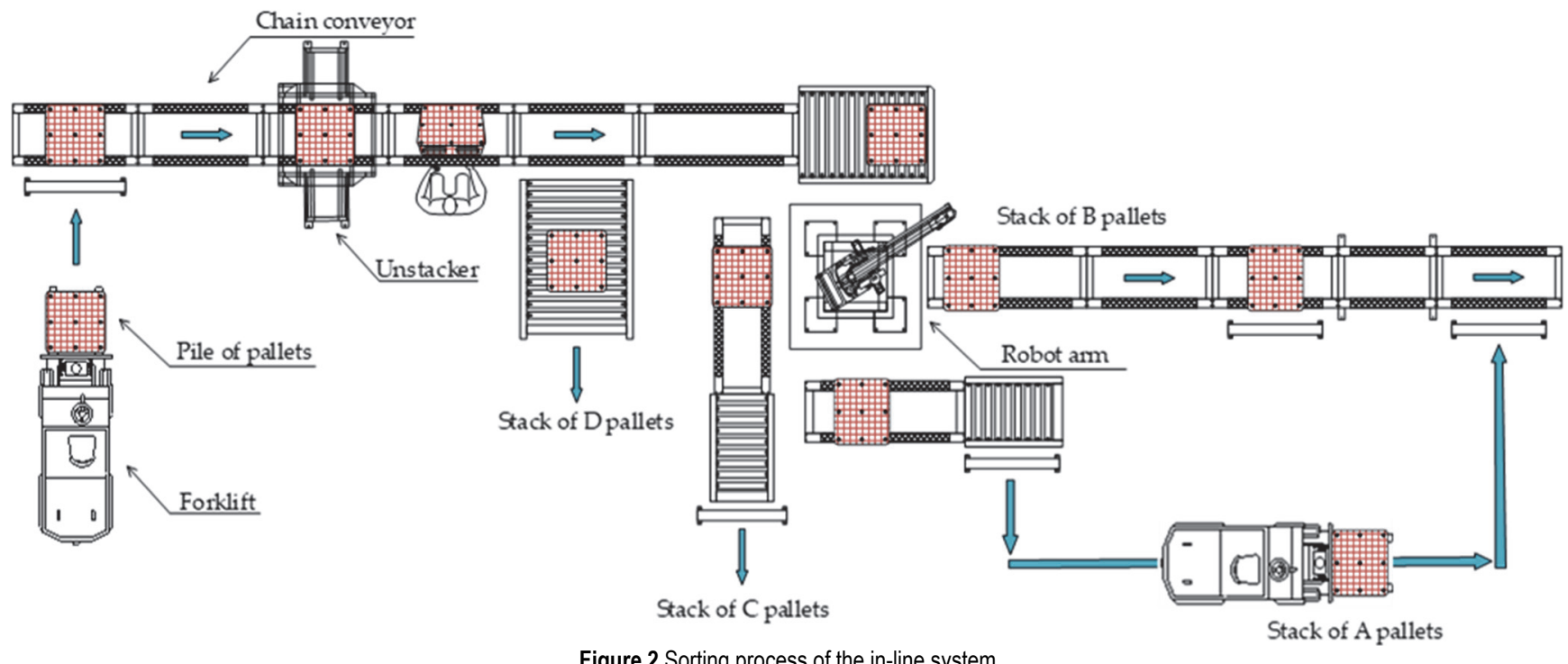

Figure 2 Sorting process of the in-line system

The main function of robot arm is to sort pallets based on their condition. However, the sorting procedure seems to not be that complicated in comparison to the robotic arm. Replacing the robotic arm with a simpler system to sort the pallets would be beneficial if the cost of the system that maintains the efficiency of the process is lower.

Queuing caused by difference in speed of machines makes the system inefficient. In the existing case study system, an average interval time between pallets (9.1 seconds) is shorter than an average spin-drying time of the spin-drying machine (10 seconds). Thus, there is currently a queue of pallets before the spin-drying part. However, despite this situation, there is one spin-drying machine in the washing process. Therefore, adding more spin-drying machines would be beneficial if the cost of another machine is lower than the costs incurred from the queuing. However, a cost-benefit analysis would need to be conducted.

\section{SIMULATION ANALYSIS}

As mentioned in Section 2, this paper used the simulation approach to design a configuration of the existing in-line system and to experiment with alternative systems. The simulation models have been set and analyzed by AutoMod software. The analytical methodology used in this research can be explained as the following sequence of the simulation approach: (1) Developing a simulation model that reflects the existing system, (2) Developing and experimenting with alternative scenarios, (3) Measuring the key performance measures, and (4) Evaluating alternatives and suggesting solution for implementation.

\section{SIMULATION EXPERIMENT}

In this section, firstly, the necessary assumptions in developing the simulation model are explained. Secondly, how to collect input data of simulation and decide the distribution of data is described. Thirdly, the simulation model reflecting the existing system is explained. Next, alternative scenarios about how to solve inefficiencies of the existing system are proposed. Lastly, the experimental design which consists of what are the main factors considered and decision of whether they have a significant effect is described.

\subsection{Assumptions and Parameters of the Simulation}

All data used in this paper is from the case study organization. The necessary assumptions have come from interviews with the company's employee and data files received by the employee. Thus, when building the simulation model, the following assumptions were used.

\subsubsection{Assumptions}

- There is no sudden failure of machines in the in-line system.

- All the pallets are identical.

\subsubsection{Parameters}

- The in-line system of the warehouse operates 20 hours a day.

- The pallet measures are $1100 \times 1100 \times 150 \mathrm{~mm}$.

- The batch size of pallets is 16 pallets.

- Each ratio of pallet class is A - 19\%; B - 74\%; C - 6\%; D - $1 \%$.

- The ratio of pallet with contaminants is $20 \%$ of all pallets.

- The worker determining the class works for 55 minutes cycles with break times of 5 minutes.

- The average sorting work time of the worker per a pallet is 3 seconds.

- There are 3 washing machines, 1 spin-drying machine, and 2 drying machines.

- The average unstacking time of the unstacker per a pallet is 9.1 seconds.

- The average spin-drying work time of spin-drying machine per a pallet is 10 seconds.

- The average stacking time of the stacker per a pallet is 7 seconds. 


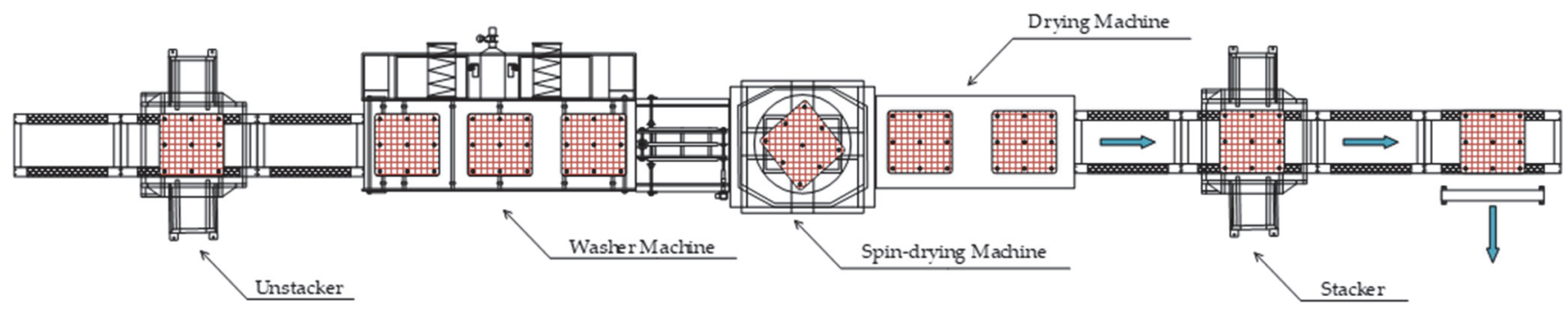

Figure 3 Washing process of the in-line system

- The average throughput of the unstacker in the sorting process is 305 pallets $/ \mathrm{hr}$.

- The length of each simulation run is 20 hours, and the warm-up time is 1 hour.

- The simulation model is run for 30 independent replications.

- The transfer time for entering the machine/process is 1 seconds.

- The velocity at which the forklift moves is 1.5 meters per second.

- The pick-up/drop-off times of the forklift are 15 seconds.

- The velocity of the conveyor varies from section to section - 15.3, 14.6, 36.6, 10.9, 36.7, 24.3 meters a second.

\subsection{Data Collection and Data Analysis}

As previously mentioned, the necessary data to develop the simulation model is from the case study company. In fact, this collected data is not an actual data, but rather the distribution data of real data from the files received by an employee of the company. The distribution data of the real data is described in the assumptions. The distribution data of input data (i.e., time data) used in process of the simulation model is summarized in Tab. 1.

Table 1 Distributions of the time data in the model of current system

\begin{tabular}{|c|c|}
\hline The unstacking time of the unstacker & $\operatorname{TRI}^{*}(8.0,9.1,10.5)$ \\
\hline $\begin{array}{c}\text { The sorting work time of the worker } \\
\text { worker }\end{array}$ & $\operatorname{EXP}^{* *}(3.0)$ \\
\hline $\begin{array}{c}\text { The removing contaminants time of the } \\
\text { machine }\end{array}$ & $\operatorname{EXP}^{* *}(5.32)$ \\
\hline $\begin{array}{c}\text { The stacking time of the stacker } \\
\text { TRI }\end{array}$ & $\operatorname{TRI}^{*}(9.2,10.0,10.4)$ \\
\hline
\end{tabular}

Triangular distribution

${ }^{* *}$ Exponential distribution

\subsection{Model Development}

The existing system mentioned in Section 3 was simulated in AutoMod software so as to analyze its main output factors like the utilization of each machine in the system, the average throughput of the system, and the average waiting time of spin-drying machine as shown in Fig. 4.

Waiting time is considered as unnecessary time consuming for productivity. Specially in in-line system, sometimes it could be considered as a "block factor" which hinders the flow of material. Thus, minimizing average waiting time could reduce the change of slowdown in the flow.

\subsection{Alternative Scenarios}

Four alternative scenarios which could help in minimizing the inefficiency of the system and improving the performance of the in-system are concisely explained below.

Scenario 1 - Adjust batch size of pallet stack: There is no modification of the model in scenario, but an adjustment of the number of the batch size. The current batch size of pallet stack is 16 . In fact, the maximum number of the batch size is 16 . Thus, it is possible to reduce this number. This scenario focuses on how the performance of the model changes when the batch size of pallets is adjusted.

Scenario 2 - Eliminate the robotic arm and change the order of process: Modification is made by replacing the robotic arm with two sorters on the chain conveyor. Moreover, the sorting work before washing process sorts only C class pallets, while class A and B pallet sorting continues after the washing process. The primary focus of this scenario is to minimize the cost inefficiency and eliminate unnecessary movements which are unstacking and stacking functions.

Scenario 3 - Add more spin-drying machines: Due to slow operating time of a spin-drying machine, there is a queue before the spin-drying machine. Therefore, modification is progressed by adding a spin-drying machine in the system to eliminate the queue. The main point of this scenario is to eliminate the queue and improve the performance of the system.

Scenario 4 - Scenario 2 \& Scenario 3: Scenario 4 is a combination of Scenario 2 and Scenario 3, which means this alternative model is constructed by applying scenario 2 and scenario 3 at the same time from the model of existing system. The point of this scenario is to confirm whether applying two ideas simultaneously is better than applying each process individually.

\subsection{Experimental Design}

Kelton [25] describes that it is significant to not only identify relevant input and output measures but experiment with simulation and understand the purpose of the project. The several alternative scenarios designed in this paper made the base of the experimental design [26]. The significant factors considered were (1) removal of constrictions or queues, (2) minimizing the total flows, and (3) availability of resources. Whether these factors were effective or not was measured by the following output measures: (1) resource utilization, (2) average throughput based on the pallet class - number of pallets per hour, and (3) the average wait time of spin-drying machine. 


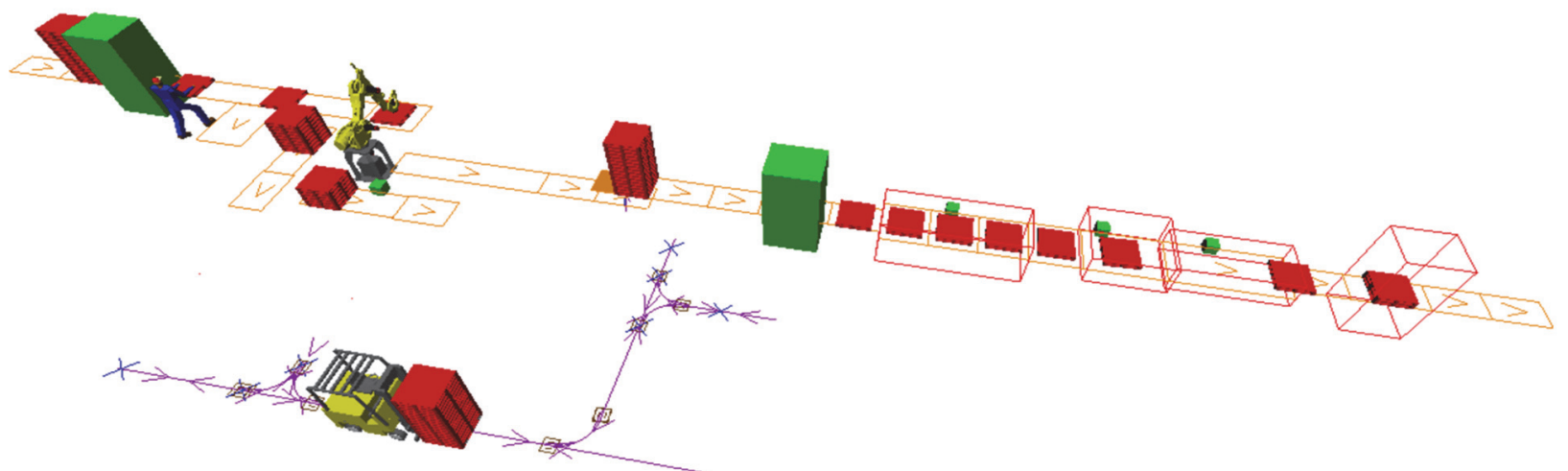

Figure 4 The simulation model for existing in-line system

\section{$6 \quad$ RESULTS AND DISCUSSION}

The simulations of existing system and alternative scenarios were conducted with an Intel(R) Core (TM) i57200U CPU with $2.50 \mathrm{GHz}$ speed and 16 GB RAM. Before executing the simulation models, as mentioned in experimental design, this research selected the throughputs based on class of pallet and the utilization of the resources as output measures. Thus, after the experiments were finished, the output measures came out. The simulation models were run for 20 hours a day. The models were replicated 30 times where each replication represents 20 hour in a day. This section explains and analyses the results of the simulation of the existing system after verifying and validating the model of the system. Next, the results of the alternative scenarios are analyzed by comparing the model of existing system with the alternative models, and then they are evaluated.

\subsection{Verification and Validation of the Model}

If a simulation model is constructed, it is necessary that the model is validated and checked for accuracy. Verification has to be done with building the model properly. It makes a comparison between the real-world system and the simulated model of the real-world system. After the model has been developed, its behavior was verified visually by running the AutoMod to be as close as possible to the current system. Then, it was validated by comparing an output factor of the model with its real value received from the employee. The average throughput per hour of the unstacker in sorting process is selected. The average obtained from 30 replications and the average measure of the real system are shown in Tab. 2. Tab. 2 indicates that percentage error of the simulation model is about 3\% and the model of existing in-system is not statistically different from the real system considering approximately a $97 \%$ level of confidence. In other words, the simulation model is valid at about the $95 \%$ level of confidence.

Table 2 Throughput per hour of unstacker in sorting process

\begin{tabular}{|c|c|c|c|}
\hline Class & Real data & Simulation model & Error $/ \%$ \\
\hline A & 57.95 & 56.30 & 2.85 \\
\hline B & 225.7 & 218.75 & 3.08 \\
\hline C & 18.3 & 17.74 & 3.06 \\
\hline D & 3.05 & 2.932 & 3.87 \\
\hline Total & 305 & 295.73 & 3.04 \\
\hline
\end{tabular}

Having run the model, the throughputs of the model of existing system based on the class of pallets were counted and are shown inTab. 3. Results in Tab. 3 indicate that the standard deviation values of all classes are very low; they are from about 0.4 to about 2.0, and the difference between maximum and minimum value of each class is also small. It means that the variation of the throughput values is tight.

Table 3 Throughput of the model of existing system (pallets/hr)

\begin{tabular}{|c|c|c|c|c|c|}
\hline Class & Avg & Std & Min & Max & Med \\
\hline A & 56.30 & 1.62 & 52.45 & 60.3 & 58.63 \\
\hline B & 218.75 & 1.95 & 212.95 & 223.15 & 220.43 \\
\hline C & 17.74 & 0.85 & 16.05 & 19.55 & 18.33 \\
\hline D & 2.93 & 0.39 & 2.2 & 3.9 & 3.28 \\
\hline Total & 295.73 & 0.87 & 292.85 & 297.85 & 296.45 \\
\hline
\end{tabular}

In addition, each utilization of the resources in the model of existing system also was shown in Tab. 5 .

Table 5 Utilization of the resources in the model of existing system

\begin{tabular}{|c|c|c|c|c|c|}
\hline Resource & Avg. & Std. & Min. & Max. & Med. \\
\hline Robot arm & 0.4601 & 0.0014 & 0.457 & 0.464 & 0.462 \\
\hline $\begin{array}{c}\text { Washing } \\
\text { Machine }\end{array}$ & 0.9390 & 0.0044 & 0.931 & 0.952 & 0.945 \\
\hline $\begin{array}{c}\text { Spin-drying } \\
\text { Machine }\end{array}$ & 0.9489 & 0.00399 & 0.943 & 0.960 & 0.954 \\
\hline $\begin{array}{c}\text { Drying } \\
\text { Machine }\end{array}$ & 0.8810 & 0.00392 & 0.875 & 0.892 & 0.886 \\
\hline
\end{tabular}

First of all, the average utilization of the robotic arm in the sorting process dips below 0.5 , and that measure is low to affect the throughput. In other words, the robotic arm does not affect the throughput of the system and does not cause the inefficiency in the system flow. In contrast, the average utilization of resources in the washing process is more than 0.88 , which is much higher than the sorting process. This is because there is a queue at the spin-drying part, which means as soon as the spin-drying machine processes a pallet, the machine starts to work again without intervals, so the utilization increases. Nevertheless, the reason the utilization does not exceed 1 is because the machine processes a stack of pallets before the next one comes, and there is a time interval. However, the utilization of resources in the washing process is too high. Hence, it needs to be lowered.

Furthermore, as the result of simulation experiment, the average waiting time of spin-drying machine is measured as 11.834 seconds. This means that there is a constriction in the spin-drying part. As can be seen, the simulation about the existing system ultimately verifies the problems of the system through the output measures selected. 
Table 4 Throughput and utilization of resources with batch size adjustment

\begin{tabular}{|c|c|c|c|c|c|c|c|}
\hline & 10 & 11 & 12 & 13 & 14 & 15 & 16 \\
\hline Throughput (pallets/hr) & 285.65 & 287.77 & 290.1 & 291.95 & 293.13 & 294.35 & 295.73 \\
\hline Utilization of robot arm & 0.4443 & 0.4477 & 0.4511 & 0.4539 & 0.4559 & 0.4581 & 0.4601 \\
\hline Utilization of washing machine & 0.9002 & 0.9100 & 0.9187 & 0.9242 & 0.9305 & 0.9345 & 0.9390 \\
\hline Utilization of spin-drying machine & 0.9171 & 0.9244 & 0.9313 & 0.9359 & 0.9414 & 0.9451 & 0.9489 \\
\hline Utilization of drying machine & 0.8506 & 0.8577 & 0.8643 & 0.8687 & 0.8739 & 0.8774 & 0.8810 \\
\hline AWT (seconds) of spin-drying machine & 11.717 & 11.762 & 11.785 & 11.808 & 11.817 & 11.822 & 11.834 \\
\hline
\end{tabular}

\subsection{Results Analysis of Experiment about the Alternative Scenarios}

There were alternative scenarios suggested to improve the efficiency of the existing system. The output performance measures proved whether the scenarios are effective. The measures were: (1) throughput per hour of the system, (2) utilization of resources, and (3) the average waiting time of spin-drying machine (seconds). The alternative models were run based on previous experimentation settings. The outcome is summarized in Tabs. 5 to 9 . Tab. 8 shows $95 \%$ level confidence interval of the resources' utilization in the washing process based on alternative scenarios. This table indicates that the utilization of resources in alternative scenarios is different from existing system (the mean of the values is outside the 95\% confidence interval of existing system).

Scenario 1: The outcome of the Scenario 1 is shown in Tab. 4. The experimentation was conducted by increasing batch size of pallets by one from 10 to 16 . Results of the scenario 1 indicate that the smaller the batch size, the smaller the throughput of the system, the utilization of resources, and also the average waiting time (AWT) of the spin-drying machine. The difference in throughput between the size 10 and 16 is about 10 pallets/hr. Moreover, there is little change in the utilization of resources and the AWT, but they also decrease as the size decreases. Therefore, it can be concluded that the decrease of the size of pallets is not a suitable alternative scenario.

Scenario 2: In Tab. 6, the average throughput of Scenario 2 based on the class of pallet is almost the same as that of the existing system's model. It means that the robotic arm and the sorter have almost the same performance in sorting the class of pallets. Therefore, replacing the robotic arm which is relatively expensive with the conveyor sorter relieves the cost inefficiency. However, the sorting of only class $\mathrm{C}$ before washing process and class $\mathrm{A}$ and $\mathrm{B}$ after washing process cannot improve the throughput of the system, and as shown in Tab. 6.

Table 6 Average throughput (pallets/hr) of alternative scenarios
\begin{tabular}{|c|c|c|c|c|}
\hline Class & Alt. 2 & Alt. 3 & Alt. 4 & $\begin{array}{c}\text { Existing } \\
\text { System }\end{array}$ \\
\hline A & 56.243 & 56.573 & 56.202 & 56.30 \\
\hline B & 218.91 & 218.22 & 219.08 & 218.75 \\
\hline C & 17.77 & 17.53 & 17.859 & 17.74 \\
\hline D & 3.148 & 2.97 & 3.11 & 2.932 \\
\hline Total & 296.07 & 295.29 & 296.25 & 295.73 \\
\hline
\end{tabular}

Tab. 7 below, it does not make much difference in the utilization of all resources. Furthermore, in Tab. 9, average waiting time of the spin-drying machine in Scenario 2 is decreased by about 1.2 seconds. Therefore, Scenario 2 can reduce the cost, but cannot improve the existing in-line system.
Table 7 Utilization of the resources

\begin{tabular}{|c|c|c|c|c|}
\hline Resource & Alt. 2 & Alt. 3 & Alt. 4 & $\begin{array}{c}\text { Existing } \\
\text { system }\end{array}$ \\
\hline $\begin{array}{c}\text { Washing } \\
\text { Machine }\end{array}$ & 0.8756 & 0.7082 & 0.7468 & 0.9390 \\
\hline $\begin{array}{c}\text { Spin-drying } \\
\text { Machine }\end{array}$ & 0.9182 & 0.7229 & 0.8174 & 0.9489 \\
\hline $\begin{array}{c}\text { Drying } \\
\text { Machine }\end{array}$ & 0.8837 & 0.7146 & 0.8066 & 0.8810 \\
\hline
\end{tabular}

Scenario 3: The results of this alternative model indicate that the utilization rates of all machines in the washing process are lowed, and average waiting time also is zero. This is because by adding a spin-drying machine in the spin-drying part, the queue problem can be solved.

Table 8 Confidence interval (C.I) of the resources' utilization

\begin{tabular}{|c|c|c|c|c|c|c|}
\hline \multicolumn{3}{|c|}{ Resource } & Alt. 2 & Alt. 3 & Alt. 4 & $\begin{array}{c}\text { Existing } \\
\text { system }\end{array}$ \\
\hline \multirow{4}{*}{$\begin{array}{l}\text { Washing } \\
\text { Machine }\end{array}$} & \multicolumn{2}{|c|}{ Avg } & 0.8756 & 0.7082 & 0.7468 & 0.9390 \\
\hline & \multicolumn{2}{|c|}{ Std } & 0.0083 & 0.0057 & 0.0051 & 0.0044 \\
\hline & \multirow{2}{*}{$\begin{array}{c}95 \% \\
\text { C.I }\end{array}$} & Upper & 0.8786 & 0.7103 & 0.7486 & 0.9405 \\
\hline & & Low & 0.8726 & 0.7061 & 0.7450 & 0.9374 \\
\hline \multirow{4}{*}{$\begin{array}{c}\text { Spin } \\
\text {-drying } \\
\text { Machine }\end{array}$} & \multicolumn{2}{|c|}{ Avg } & 0.9182 & 0.7229 & 0.8174 & 0.9489 \\
\hline & \multicolumn{2}{|c|}{ Std } & 0.0032 & 0.0040 & 0.0044 & 0.0040 \\
\hline & \multirow{2}{*}{$\begin{array}{c}95 \% \\
\text { C.I }\end{array}$} & Upp & 0.9194 & 0.7243 & 0.8190 & 0.9503 \\
\hline & & Lower & 0.9170 & 0.7215 & 0.8158 & 0.9475 \\
\hline \multirow{4}{*}{$\begin{array}{l}\text { Drying } \\
\text { Machine }\end{array}$} & \multicolumn{2}{|c|}{ Avg } & 0.8837 & 0.7146 & 0.8066 & 0.8810 \\
\hline & \multicolumn{2}{|c|}{ Std } & 0.0041 & 0.0043 & 0.0042 & 0.0039 \\
\hline & \multirow{2}{*}{$\begin{array}{c}95 \% \\
\text { C.I }\end{array}$} & Upper & 0.8852 & 0.7161 & 0.8081 & 0.8824 \\
\hline & & Lower & 0.8822 & 0.7131 & 0.8051 & 0.8796 \\
\hline
\end{tabular}

Table 9 Average waiting time of spin-drying machine in alternative scenarios

\begin{tabular}{|c|c|c|c|}
\hline Alt. 2 & Alt. 3 & Alt. 4 & $\begin{array}{c}\text { Existing } \\
\text { system }\end{array}$ \\
\hline 10.653 & 0.0 & 0.0 & 11.834 \\
\hline
\end{tabular}

Scenario 4: The results of Scenario 4 are similar to those of Scenario 3. Scenario 4 also lowers the utilization rates of all machines in the washing process and makes the average waiting time to be zero like Scenario 3. However, the difference of them is the value of the utilization rates. In this case, the utilization rates of the washing process's resources are higher than those of Scenario 3. This is because eliminating the unnecessary processes of Scenario 2 saves the time of conducting the unnecessary processes. Therefore, the pallets are sent to the washing process faster.

\section{CONCLUSIONS}

A pallet sorting and washing process in the warehouse of a pallet polling company has several problems. The layout for this process is an in-line system or process which starts with sorting process and ends with washing process. The problems are the cost inefficiency due to the usage of a robotic arm, the unnecessary work of stacking and unstacking pallets, and the queue (i.e. the bottleneck). So far numerous studies have explored how to improve the material flow system as this in-line system, and a variety of research methods for improving the system have been 
suggested. Of different methods, a simulation approach can build the existing system, analyze the performance of the system and review whether the solutions about the existing problems are effective or not by experimenting with those solutions and comparing the model of existing system with the solution models. For these reasons, this paper used a simulation approach as the research method.

Necessary data to develop the simulation model was received by an employee of the pallet polling company. However, collected data was the distribution data of real data from the files received by the employee. Hence, the data analysis procedure was unnecessary. Moreover, the assumptions used when developing the simulation model were taken from the data.

We made the simulation model of existing in-line system using the data from the assumptions. The model was verified visually by running the model and comparing between the real-world system and the simulated model of the real-world system, and then it was validated at about 95\% level of confidence.

In this paper, 4 alternative scenarios are established to improve the research problems, and the meaningful results and effects were observed through the experiments. The experimental results showed that first, the smaller the batch size, the smaller the throughput of the system, the utilization of resources, and the average waiting time (AWT) of spin-drying machine. Secondly, in the sorting process, replacing the robot arm, which is relatively expensive, with the conveyor sorter, relieves the cost inefficiency. Thirdly, by adding a spin-drying machine in the spin-drying part, the queue problem can be solved. Combining various scenarios and implementing them will synergistically add to the desired effects of improving the process.

This research could help pallet polling companies using an in-line system as pallet sorting and washing process like the company in this paper. Not only the manufacturing systems using the in-line system, but also other systems using it would refer to this paper. Moreover, this study is worthy of a specific case of diverse in-line processes. In-line process system would not easily change its configuration because of its characteristic - equipmentbased system. Simulation model introduced in this paper could provide an insight into how to approach a problem when we need process improvement for not that flexible system.

\section{Acknowledgements}

Thanks to Network Operation Team in Korea Logis Pool CO. LTD. for their data supports.

\section{REFERENCES}

[1] Tompkins, J. A., White, J. A., Bozer, Y. A., \& Tanchoco, J. M. A. (2010). Facilities planning. John Wiley \& Sons.

[2] Hopp, W. J. \& Spearman, M. L. (2011). Factory physics. Waveland Press.

[3] Bilbao, A. M., Carrano, A. L., Hewitt, M., \& Thorn, B. K. (2011). On the environmental impacts of palletmanagement operations. Management Research Review, 34(11), 12221236. https://doi.org/10.1108/01409171111178765

[4] Roy, D., Carrano, A. L., Pazour, J. A., \& Gupta, A. (2016). Cost-effective pallet management strategies. Transportation
Research Part E: Logistics and Transportation Review, 93, 358-371. https://doi.org/10.1016/j.tre.2016.06.005

[5] Alvarez-Vargas, R., Dallery, Y., \& David, R. (1994). A study of the continuous flow model of production lines with unreliable machines and finite buffers. Journal of Manufacturing Systems, 13(3), 221-234. https://doi.org/10.1016/0278-6125(94)90006-X

[6] Dhouib, K., Gharbi, A., \& Ayed, S. (2009). Simulation based throughput assessment of non-homogeneous transfer lines. International Journal of Simulation Modelling (IJSIMM), $8(1)$.

[7] Xie, X. L. (1989). Approximate Analysis of Transfer Lines with Unreliable Machines and Finite Buffers. IEEE Transactions on Automatic Control, 34(9), 943-953. https://doi.org/10.1109/9.35807

[8] Hosseini, B. \& Tan, B. (2017). Simulation and optimization of continuous-flow production systems with a finite buffer by using mathematical programming. IISE Transactions, 49(3), 255-267. https://doi.org/10.1080/0740817X.2016.1217103

[9] Buzacott, J. A. (1971). The role of inventory banks in flowline production systems. International Journal of Production Research, 9(4), 425-436. https://doi.org/10.1080/00207547108929891

[10] Yeralan, S. \& Muth, E. J. (1987). A general model of a production line with intermediate buffer and station breakdown. IIE Transactions (Institute of Industrial Engineers), 19(2), 130-139. https://doi.org/10.1080/07408178708975379

[11] Zimmern, B. (1956). Études de la propagation des arrêts aléatoires dans les chaînes de production. Revue de Statistique Appliquée, 4(1), 85-104.

[12] Sevast'yanov, B. A. (1962). Influence of Storage Bin Capacity on the Average Standstill Time of a Production Line. Theory of Probability \& Its Applications, 7(4), 429438. https://doi.org/10.1137/1107040

[13] Wijngaard, J. (1979). The effect of interstage buffer storage on the output of two unreliable production units in series, with different production rates. AIIE Transactions, 11(1), 42-47. https://doi.org/10.1080/05695557908974399

[14] Murphy, R. A. (1975). The effect of surge on system availability. AIIE Transactions, 7(4), 439-443. https://doi.org/10.1080/05695557508975029

[15] Yeralan, S., Franck, W. E., \& Quasem, M. A. (1986). A continuous materials flow production line model with station breakdown. European Journal of Operational Research, 27(3), 289-300. https://doi.org/10.1016/0377-2217(86)90326-7

[16] Dallery, Y. (1994). On modeling failure and repair times in stochastic models of manufacturing systems using generalized exponential distributions. Queueing Systems, 15(1-4), 199-209. https://doi.org/10.1007/BF01189237

[17] Levantesi, R., Matta, A., \& Tolio, T. (2003). Performance evaluation of continuous production lines with machines having different processing times and multiple failure modes. Performance Evaluation, 51(2-4), 247-268. https://doi.org/10.1016/S0166-5316(02)00098-6

[18] Kim, J. \& Gershwin, S. B. (2005). Integrated quality and quantity modeling of a production line. OR Spectrum, 27(23), 287-314. https://doi.org/10.1007/s00291-005-0202-1

[19] Colledani, M. \& Gershwin, S. B. (2013). A decomposition method for approximate evaluation of continuous flow multi-stage lines with general Markovian machines. Annals of Operations Research, 209(1), 5-40. https://doi.org/10.1007/s10479-011-0961-9

[20] Fleuren, S., Bierbooms, R., \& Adan, I. (2014). Performance analysis of exponential multi-server production lines with fluid flow and finite buffers. Stochastic Models, 30(4), 469493. 
[21] Koenigsberg, E. (1959). Production Lines and Internal Storage - A Review. Management Science, 5(4), 410-433. https://doi.org/10.1287/mnsc.5.4.410

[22] Rohleder, T. R., Lewkonia, P., Bischak, D. P., Duffy, P., \& Hendijani, R. (2011). Using simulation modeling to improve patient flow at an outpatient orthopedic clinic. Health Care Management Science, 14(2), 135-145. https://doi.org/10.1007/s10729-010-9145-4

[23] Huang, S. H., Dismukes, J. P., Shi, J., Su, Q., Razzak, M. A., Bodhale, R., \& Robinson, D. E. (2003). Manufacturing productivity improvement using effectiveness metrics and simulation analysis. International Journal of Production Research, 41(3), 513-527. https://doi.org/10.1080/0020754021000042391

[24] Kamrani, M., Hashemi Esmaeil Abadi, S. M., \& Rahimpour Golroudbary, S. (2014). Traffic simulation of two adjacent unsignalized T-junctions during rush hours using Arena software. Simulation Modelling Practice and Theory, 49, 167-179. https://doi.org/10.1016/j.simpat.2014.09.006

[25] Kelton, W. D. (1997). Statistical analysis of simulation output. Winter Simulation Conference, 23-30.

[26] Venkatadri, V., Raghavan, V. A., Kesavakumaran, V., Lam, S. S., \& Srihari, K. (2011). Simulation based alternatives for overall process improvement at the cardiac catheterization lab. Simulation Modelling Practice and Theory, 19(7), 15441557. https://doi.org/10.1016/j.simpat.2011.04.004

\section{Contact information:}

\section{Yeongmin YUN}

School of Air Transport, Transportation and Logistics,

Korea Aerospace University,

Goyang-si, Gyeonggi-do, 10540, Korea

E-mail:yym7079@kau.kr

Junjae CHAE, PhD, Full Professor

(Corresponding author)

School of Air Transport, Transportation and Logistics,

Korea Aerospace University,

Goyang-si, Gyeonggi-do, 10540, Korea

E-mail: jchae@kau.ac.kr 